\title{
Paper Call-level Analysis of a Two-Link Multirate Loss Model based on a Convolution Algorithm
}

\author{
Stefanos G. Sagkriotis ${ }^{1}$, Spyros K. Pantelis ${ }^{2}$, Ioannis D. Moscholios ${ }^{2}$, and Vassilios G. Vassilakis ${ }^{3}$ \\ ${ }^{1}$ School of Computer Science, University of Glasgow, Glasgow, United Kingdom \\ ${ }^{2}$ University of Peloponnese, Tripolis, Greece \\ ${ }^{3}$ University of York, York, United Kingdom
}

https://doi.org/10.26636/jtit.2018.123217

\begin{abstract}
We consider a two-link system that accommodates Poisson arriving calls from different service-classes and propose a multirate teletraffic loss model for its analysis. Each link has two thresholds, which refer to the number of inservice calls in the link. The lowest threshold, named support threshold, defines up to which point the link can support calls offloaded from the other link. The highest threshold, named offloading threshold, defines the point where the link starts offloading calls to the other link. The adopted bandwidth sharing policy is the complete sharing policy, in which a call can be accepted in a link if there exist enough available bandwidth units. The model does not have a product form solution for the steady state probabilities. However, we propose approximate formulas, based on a convolution algorithm, for the calculation of call blocking probabilities. The accuracy of the formulas is verified through simulation and found to be quite satisfactory.
\end{abstract}

Keywords—call blocking, convolution, loss model, offloading, product form.

\section{Introduction}

Quality of service (QoS) mechanisms are necessary in contemporary communication networks in order to provide the required bandwidth needed by calls. In the case of call-level traffic in a single link, modeled as a loss system, such a QoS mechanism is a bandwidth sharing policy [1]. The simplest bandwidth sharing policy is the complete sharing (CS) policy, where a new call is accepted in the system if there exist enough available bandwidth units (b.u.). Otherwise, call blocking occurs. The simplest teletraffic loss model that adopts the CS policy is the classic Erlang model [1]. In this model, the call arrival process is Poisson, while each call requires one b.u. to be accepted in the system. An accepted call has a generally distributed service time. The fact that call blocking probabilities (CBP) are calculated via the classic Erlang B formula has led to numerous extensions of Erlang's model for the call-level analysis of wired (e.g. [2]-[16]), wireless (e.g. [17]-[28]), satellite (e.g. [29]-[31]) and optical networks (e.g. [32]-[37]).

In the work of [25], the Erlang B formula has been adopted for the determination of CBP in a two access link sys- tem that accommodates Poisson arriving calls of a single service-class. Each access link is modelled as a loss system (i.e. no queueing is permitted) and has two thresholds, which refer to the number of in-service calls in the link. The lowest threshold, named support threshold, defines up to which point the access link can support calls offloaded from the other access link. The highest threshold, named offloading threshold, defines the point where the access link starts offloading calls to the other access link. By the term offloaded call, we refer to a call that initially arrived in a link, but is served by the other link, if there exist available b.u. The model of [25] does not have a product form solution (PFS) for the steady state probabilities. This is due to the fact that the offloading mechanism destroys local balance (LB) between adjacent states (states that differ only by one call) of the system. To calculate the various performance measures of the system, e.g. CBP or link utilization, either a linear system of global balance (GB) equations should be solved or an approximate method that relies on the independence between the links and the classic Erlang B formula can be adopted. The system of GB equations leads to an accurate calculation of the performance measures but it requires the knowledge of the state space of the two-link system. Such a state space may consist of millions of states if the capacity of the links is high. Thus, the method of solving the GB equations can only be applied in small (tutorial) systems [38]. On the other hand, the link independence assumption and the Erlang B formula facilitate the necessary calculations.

A potential application of the offloading scheme of [25] is in the area of mobile/Wi-Fi networks. To manage the increasing traffic in mobile networks, traffic can be offloaded to Wi-Fi networks [39], [40]. To further increase the available bandwidth of Wi-Fi access links, recent research focuses on the aggregation of backhaul access link capacities and on the bandwidth sharing policies that should be adopted (see e.g. the BeWi-Fi concept that enables users in proximity to share their Internet access if their link utilization is below a threshold) [41]. The impact of such an aggregation to CBP in the case of a single service-class can be well studied by the offloading scheme of [25]. 
In this paper, we extend the model of [25] to include the important case of multirate traffic, i.e. we consider a two access link system that accommodates Poisson arriving calls of different service-classes and different bandwidth-per-call requirements. The CBP calculation in the proposed two-link model under the CS policy is based on the classic Erlang Multirate Loss Model (EMLM) [42], [43], which refers to a single link. To differentiate, we name the proposed model 2EMLM. In the 2EMLM model, the determination of CBP can be done via a 3-step convolution algorithm. The latter exploits the PFS of the EMLM and the principle of independency among service-classes and, therefore, the link occupancy distribution can be determined by successively convolving the link occupancy distributions obtained for each service-class. Contrary to macro-state recursive formulas (such as the classic Kaufman-Roberts formula used for CBP calculation in EMLM [42], [43]), a convolution algorithm keeps the micro-state information of the number of in-service calls in a link. Such information is necessary when studying more complicated (than the CS policy) call admission policies (e.g. [44]-[53]).

The remainder of this paper is organized as follows: in Section 2, we review the system of [25]. In Section 3, we propose the 2EMLM and provide a convolution algorithm for CBP determination. In Section 4, we provide analytical and simulation CBP results for the proposed model. We conclude in Section 5. In the Appendix, we provide a tutorial example of the system of [25].

\section{The Two-Link System with Single-Rate Traffic}

We consider a system of two links with capacities $C_{1}$ and $C_{2}$ b.u., respectively. Each link accommodates Poisson arriving calls of a single service-class which require one b.u. in order to be connected in a link. Let $\lambda_{1}$ and $\lambda_{2}$ be the arrival rates in the 1 st and the 2 nd link, respectively. We also denote by $j_{1}$ and $j_{2}$ the occupied b.u. in the 1 st and the 2nd link, respectively. Then, $0 \leq j_{1} \leq C_{1}$ and $0 \leq j_{2} \leq C_{2}$. Since calls require one b.u., the values of $j_{1}, j_{2}$ also represent the number of in-service calls in the 1 st and the 2 nd link, respectively.

Each link $l(l=1,2)$ has two different thresholds: the support threshold $t h_{1 l}$ and the offloading threshold $t h_{2 l}$, with $t h_{1 l}<t h_{2 l}$ and $0 \leq t h_{1 l}, t h_{2 l} \leq 1$. Assuming that $\lfloor x\rfloor$ is the largest integer not exceeding $x$, the role of these thresholds, in the $l$-th link, is the following (see Fig. 1):

- If $0 \leq j_{l}<\left\lfloor t h_{1 l} C_{l}\right\rfloor$ then the $l$-th link is in a support mode of operation, i.e. it accepts and serves not only new calls that initially arrive in the $l$-th link, but also new calls offloaded from the $m$-th link $(m=1,2, m \neq l)$.

- If $\left\lfloor t h_{1 l} C_{l}\right\rfloor \leq j_{l}<\left\lfloor t h_{2 l} C_{l}\right\rfloor$ then the $l$-th link is in a normal mode of operation, i.e. it does not accept calls offloaded from the $m$-th link. It only accepts calls that initially arrive in the $l$-th link.

- If $\left\lfloor t h_{2 l} C_{l}\right\rfloor \leq j_{l}$ then the $l$-th link is in an offloading mode of operation, i.e. a new call that initially arrives in the $l$-th link will be offloaded to the $m$-th link. If the $m$-th link is in support mode (i.e. $0 \leq j_{m}<$ $\left\lfloor t h_{1 m} C_{m}\right\rfloor$ ) then the call will be accepted in the $m$-th link. If the $m$-th link is not in support mode and $j_{l} \leq C_{l}-1$, the call will be accepted in the $l$-th link. Otherwise the call will be blocked and lost.

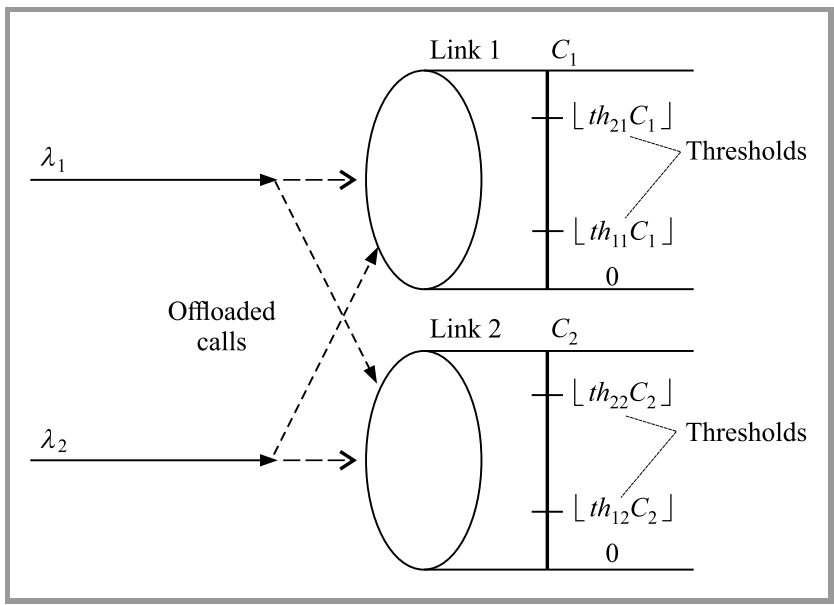

Fig. 1. The system of the two links.

Based on the above, the admission of a new call that initially arrives in the $l$-th link $(l=1,2)$ is summarized in the following steps:

1) If $\left(0 \leq j_{l}<\left\lfloor t h_{2 l} C_{l}\right\rfloor\right)$ then the call is accepted by the $l$-th link and remains for a generally distributed servicetime with mean $\mu^{-1}$.

2) If $\left\lfloor t h_{2 l} C_{l}\right\rfloor \leq j_{l}$ then:

2a) if $0 \leq j_{m}<\left\lfloor t h_{1 m} C_{m}\right\rfloor$ the call is offloaded to the $m$-th link and remains for a generally distributed service-time with mean $\mu^{-1}$;

2b) if $\left\lfloor t h_{1 m} C_{m}\right\rfloor \leq j_{m}$, the $m$-th link is in a normal mode of operation and does not support offloaded calls from the $l$-th link. In that case, the call will try to be accepted in the $l$-th link. If $j_{l} \leq C_{l}-1$, then the call is accepted in the $l$-th link and remains for a generally distributed service-time. Otherwise, the call is blocked and lost without further affecting the system of the two links.

A tutorial example in the Appendix, presents in detail the call admission mechanism and the required calculations for CBP determination.

Due to the support and offloading modes of operation of the two links, the 2-D Markov chain of the system is not reversible and, therefore, LB between adjacent states (states that differ only by one call) is destroyed. Thus, the steady 
state distribution, $P(j)=P\left(j_{1}, j_{2}\right)$, of this system cannot be described by a PFS. To determine the values of $P\left(j_{1}, j_{2}\right)$ (and consequently CBP) there exist two different methods. The first method provides accurate results (compared to simulation) but requires the knowledge of the state space of the system and the solution of the set of linear GB equations for each state $j=\left(j_{1}, j_{2}\right)$ expressed as rate into state $j=$ rate out of state $j$ :

$$
\begin{aligned}
& \lambda_{1}\left(j_{1}-1, j_{2}\right) P\left(j_{1}-1, j_{2}\right)+\lambda_{2}\left(j_{1}, j_{2}-1\right) P\left(j_{1}, j_{2}-1\right)+ \\
& +\left(j_{1}+1\right) \mu P\left(j_{1}+1, j_{2}\right)+\left(j_{2}+1\right) \mu P\left(j_{1}, j_{2}+1\right)= \\
& =\lambda_{1}\left(j_{1}, j_{2}\right) P\left(j_{1}, j_{2}\right)+\lambda_{2}\left(j_{1}, j_{2}\right) P\left(j_{1}, j_{2}\right)+ \\
& +\left(j_{1} \mu+j_{2} \mu\right) P\left(j_{1}, j_{2}\right)
\end{aligned}
$$

where:

$$
\lambda_{1}\left(j_{1}, j_{2}\right) \stackrel{l=1,2, m \neq l}{=} \begin{cases}\lambda_{l}+\lambda_{m}, & \text { if }\left(j_{l}<\left\lfloor t h_{1 l} C_{l}\right\rfloor\right) \\ & \cap\left(j_{m} \geq\left\lfloor t h_{2 m} C_{m}\right\rfloor\right) \\ 0, & \text { if }\left(j_{l} \geq\left\lfloor t h_{2 l} C_{l}\right\rfloor\right) \\ & \cap\left(j_{m}<\left\lfloor t h_{1 m} C_{m}\right\rfloor\right) . \\ 0, & \text { if }\left(j_{1}, j_{2}\right) \text { is } \\ & \text { a boundary state } \\ \lambda_{l}, & \text { otherwise }\end{cases}
$$

Having obtained the values of $P\left(j_{1}, j_{2}\right)$, we can determine the CBP in the 1 st and the 2 nd link, $P_{b_{1}}^{\prime}$ and $P_{b_{2}}^{\prime}$ via Eqs. (3) and (4), respectively [25]:

$$
\begin{aligned}
& P_{b_{1}}^{\prime}=\sum_{j_{2}=\left\lfloor t h_{12} C_{2}\right\rfloor}^{C_{2}} P\left(C_{1}, j_{2}\right), \\
& P_{b_{2}}^{\prime}=\sum_{j_{1}=\left\lfloor t h_{11} C_{1}\right\rfloor}^{C_{2}} P\left(j_{1}, C_{2}\right) .
\end{aligned}
$$

In addition, we can calculate the total blocking probability in the system via the following weighted summation:

$$
P_{b}^{\prime}=\frac{\lambda_{1}}{\lambda_{1}+\lambda_{2}} P_{b_{1}}^{\prime}+\frac{\lambda_{2}}{\lambda_{1}+\lambda_{2}} P_{b_{2}}^{\prime} .
$$

Before we proceed with the second method, we emphasize that the state space determination and the solution of the set of GB equations can be quite complex even for systems of moderate size and, therefore, is only practically used for small tutorial examples (see Appendix).

The second method provides approximate CBP results by assuming that the two links operate independently from one another. Such an assumption simplifies the necessary CBP calculations. Since each independent link behaves as an Erlang loss system, the CBP in the 1st and the 2nd link can be approximated by Eqs. (6) and (7), respectively:

$$
\begin{aligned}
& P_{b_{1}}=P_{1}\left(C_{1}\right) P_{2}\left(j_{2} \geq\left\lfloor t h_{12} C_{2}\right\rfloor\right), \\
& P_{b_{2}}=P_{2}\left(C_{2}\right) P_{1}\left(j_{1} \geq\left\lfloor t h_{11} C_{1}\right\rfloor\right),
\end{aligned}
$$

where $P_{l}\left(C_{l}\right)$ refers to the $\mathrm{CBP}$ in the $l$-th link $(l=1,2)$ which can be determined by the Erlang B formula:

$$
P_{l}\left(C_{l}\right)=\frac{\frac{\alpha_{l}^{C_{l}}}{C_{l} !}}{\sum_{i=0}^{C_{l}} \frac{\alpha_{l}^{i}}{i !}}, \quad \alpha_{l}=\frac{\lambda_{l}}{\mu}
$$

As far as the values of $P_{l}\left(j_{l} \geq\left\lfloor t h_{1 l} C_{l}\right\rfloor\right)$ are concerned they are given by:

$$
P_{l}\left(j_{l} \geq\left\lfloor t h_{1 l} C_{l}\right\rfloor\right)=\sum_{j_{l}=\left\lfloor t h_{1 l} C_{l}\right\rfloor}^{C_{l}} P_{l}\left(j_{l}\right),
$$

where $P_{l}\left(j_{l}\right)$ is determined by the truncated Poisson distribution:

$$
P_{l}\left(j_{l}\right)=\frac{\frac{\alpha_{l}^{j_{l}}}{j_{l} !}}{\sum_{i=0}^{C_{l}} \frac{\alpha_{l}^{i}}{i !}}, \quad \alpha_{l}=\frac{\lambda_{l}}{\mu} .
$$

The rationale behind Eqs. (6) and (7) is that a call that initially arrives in the $l$-th link will be blocked if there are no available b.u. in that link and the $m$-th link is not in support mode of operation.

Finally, the total blocking probability can be determined via the following formula:

$$
P_{b}=\frac{\lambda_{1}}{\lambda_{1}+\lambda_{2}} P_{b_{1}}+\frac{\lambda_{2}}{\lambda_{1}+\lambda_{2}} P_{b_{2}} .
$$

\section{The Proposed 2EMLM}

In the proposed 2EMLM, we consider again the system of the two links. Each link accommodates Poisson arriving calls of $K$ service-classes. Calls of service-class $k$ ( $k=$ $1, \ldots, K)$ require $b_{k}$ b.u. in order to be connected in a link. Let $\lambda_{1 k}$ and $\lambda_{2 k}$ be the arrival rates in the 1 st and the 2 nd link of service-class $k$ calls, respectively. We also denote by $j_{1}$ and $j_{2}$ the occupied b.u. in the 1 st and the 2 nd, respectively. Then, $0 \leq j_{1} \leq C_{1}$ and $0 \leq j_{2} \leq C_{2}$. Similar to Section 2, each link $l(l=1,2)$ has a support threshold $t h_{1 l}$ and an offloading threshold $t h_{2 l}$, with $t h_{1 l}<t h_{2 l}$ and $0 \leq t h_{1 l}, t h_{2 l} \leq 1$.

The call admission of a new service-class $k$ call that initially arrives in the $l$-th link $(l=1,2)$ is summarized in the following steps:

1) If $\left(0 \leq j_{l}<\left\lfloor t h_{2 l} C_{l}\right\rfloor\right) \cap\left(j_{l}+b_{k} \leq C_{l}\right)$ then the call is accepted by the $l$-th link and remains for a generally distributed service-time with mean $\mu_{k}^{-1}$.

2) If $\left\lfloor t h_{2 l} C_{l}\right\rfloor \leq j_{l}$ then:

2a) if $\left(0 \leq j_{m}<\left\lfloor t h_{1 m} C_{m}\right\rfloor\right) \cap\left(j_{m}+b_{k} \leq C_{m}\right)$ the call is offloaded to the $m$-th link and remains for a generally distributed service-time with mean $\mu_{k}^{-1}$;

2b) if $\left\lfloor t h_{1 m} C_{m}\right\rfloor \leq j_{m}$, the $m$-th link is in normal mode of operation and does not support offloaded calls from the $l$-th link. In that case, the call will try to 
be accepted in the $l$-th link. If $j_{l}+b_{k} \leq C_{l}$, then the call is accepted in the $l$-th link and remains for a generally distributed service-time with mean $\mu_{k}^{-1}$. Otherwise, the call is blocked and lost.

To determine in an approximate but efficient way the CBP of service-class $k$ calls we assume that the two links operate independently from one another. In that case, each independent link behaves as an EMLM system, and therefore the CBP of service-class $k$ calls in the 1 st and the 2 nd link can be approximated by Eqs. (12) and (13), respectively:

$$
\begin{aligned}
& P_{b_{1 k}}=P_{1 k}\left(C_{1}\right) P_{2}\left(j_{2} \geq\left\lfloor t h_{12} C_{2}\right\rfloor\right), \\
& P_{b_{2 k}}=P_{2 k}\left(C_{2}\right) P_{1}\left(j_{1} \geq\left\lfloor t h_{11} C_{1}\right\rfloor\right),
\end{aligned}
$$

where $P_{l k}\left(C_{l}\right)$ refers to the CBP of service-class $k$ calls in the $l$-th link $(l=1,2)$.

The values of $P_{l k}\left(C_{l}\right)$ in Eqs. (12) and (13) are determined by:

$$
P_{l k}\left(C_{l}\right)=\sum_{j_{l}=C_{l}-b_{k}+1}^{C_{l}} G_{l}^{-1} q\left(j_{l}\right),
$$

where $q\left(j_{l}\right)$ refers to the unnormalized values of the link occupancy distribution of link $l(l=1,2)$ while $G_{l}=\sum_{j_{l}=0}^{C_{l}} q\left(j_{l}\right)$ is the normalization constant.

In Eq. (14), the values of $q\left(j_{l}\right)$ can be recursively determined via a 3 -step convolution algorithm. To describe it, let $q_{l, k}(j)(k=1, \ldots, K)$ be the link occupancy distribution assuming that only service-class $k$ exists in the link $l$. Then, the 2EMLM convolution algorithm is as follows:

Step 1. Determine $q_{l, k}(j)$ of each service-class $k$ via:

$$
q_{l, k}\left(j_{l}\right)=q_{l, k}(0) \frac{\alpha_{l k}^{i}}{i !}, \text { for } 1 \leq i \leq\left\lfloor\frac{C_{l}}{b_{k}}\right\rfloor \text { and } j_{l}=i \times b_{k},
$$

where $\alpha_{l k}=\lambda_{l k} / \mu_{k}$ is the offered traffic-load (in Erl) of service-class $k$ calls in link $l$.

Step 2. Determine the aggregated occupancy distribution $Q_{(-k)}$ based on the successive convolution of all serviceclasses (in link $l$ ) apart from service-class $k$ :

$$
Q_{(-k)}=q_{l, 1} \cdot \ldots \cdot q_{l, k-1} \cdot q_{l, k+1} \cdot \ldots \cdot q_{l, K} .
$$

The term "successive" means that initially we convolve $q_{l, 1}$ and $q_{l, 2}$ to obtain $q_{l, 12}$. Then we convolve $q_{l, 1}$ with $q_{l, 3}$ to obtain $q_{l, 123}$ etc. The convolution operation between service-classes $k$ and $r$ is as follows:

$$
\begin{aligned}
& q_{l, k} \cdot q_{l, r}=\left\{q_{l, k}(0) q_{l, r}(0), \sum_{x=0}^{1} q_{l, k}(x) q_{l, r}(1-x), \ldots,\right. \\
& \left.\ldots, \sum_{x=0}^{C} q_{l, k}(x) q_{l, r}\left(C_{l}-x\right)\right\} .
\end{aligned}
$$

Step 3. Determine the values of $q\left(j_{l}\right)$ based on the convolution operation of $Q_{l,(-k)}$ (step 2) and $q_{l, k}$ as follows:

$$
\begin{aligned}
& Q_{l,(-k)} \cdot q_{l, k}=\left\{Q_{l,(-k)}(0) q_{l, k}(0), \sum_{x=0}^{1} Q_{l,(-k)}(x)\right. \\
& \left.q_{l, k}(1-x), \ldots, \sum_{x=0}^{C_{l}} Q_{l,(-k)}(x) q_{l, k}\left(C_{l}-x\right)\right\} .
\end{aligned}
$$

Normalizing the values of (18), we obtain the occupancy distribution $q\left(j_{l}\right), j=0,1, \ldots, C_{l}$ via the formulas:

$$
\begin{gathered}
q(0)=\frac{Q_{l,(-k)}(0) q_{l, k}(0)}{G_{l}} \\
q(j)=\frac{\sum_{x=0}^{j} Q_{l,(-k)}(x) q_{l, k}(j-x)}{G_{l}}, j=1, \ldots, C_{l}
\end{gathered}
$$

As far as the values of $P_{l}\left(j_{l} \geq\left\lfloor t h_{1 l} C_{l}\right\rfloor\right)$, in Eqs. (12) and (13), are concerned, they are calculated by:

$$
P_{l}\left(j_{l} \geq\left\lfloor t h_{1 l} C_{l}\right\rfloor\right)=\sum_{j_{l}=\left\lfloor t h_{1 l} C_{l}\right\rfloor}^{C_{l}} G_{l}^{-1} q\left(j_{l}\right),
$$

where $q\left(j_{l}\right)$ is determined via (19).

Finally, we propose the following formula for the total blocking probability of service-class $k$ calls in the system of the two links:

$$
P_{b_{k}}=\frac{\lambda_{1 k}}{\lambda_{1 k}+\lambda_{2 k}} P_{b_{1 k}}+\frac{\lambda_{2 k}}{\lambda_{1 k}+\lambda_{2 k}} P_{b_{2 k}}
$$

\section{Numerical Examples - Evaluation}

In this section, we present an application example and provide analytical and simulation results of the total CBP of the proposed model. Simulation results are derived via the Simscript III simulation language [54] and are mean values of 7 runs. As far as the reliability ranges are concerned, they are less than two orders of magnitude, and therefore are not presented in the following figures. All simulation runs are based on the generation of eight million calls per

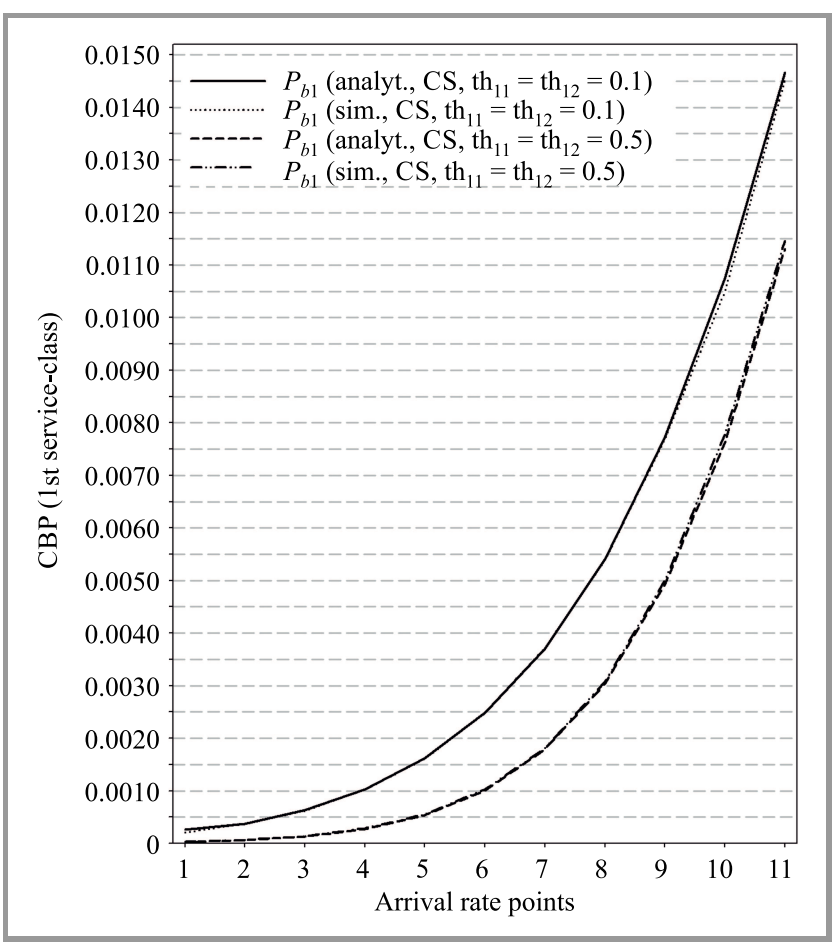

Fig. 2. CBP under the CS policy - 1st service-class. 


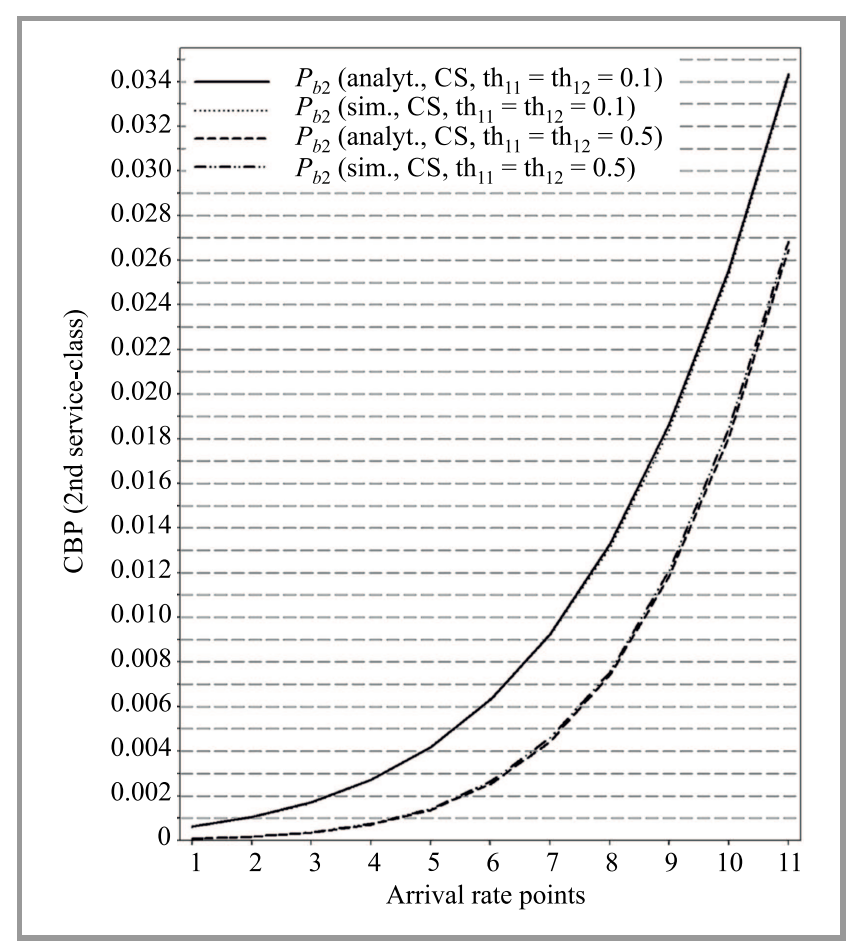

Fig. 3. CBP under the CS policy - 2nd service-class.

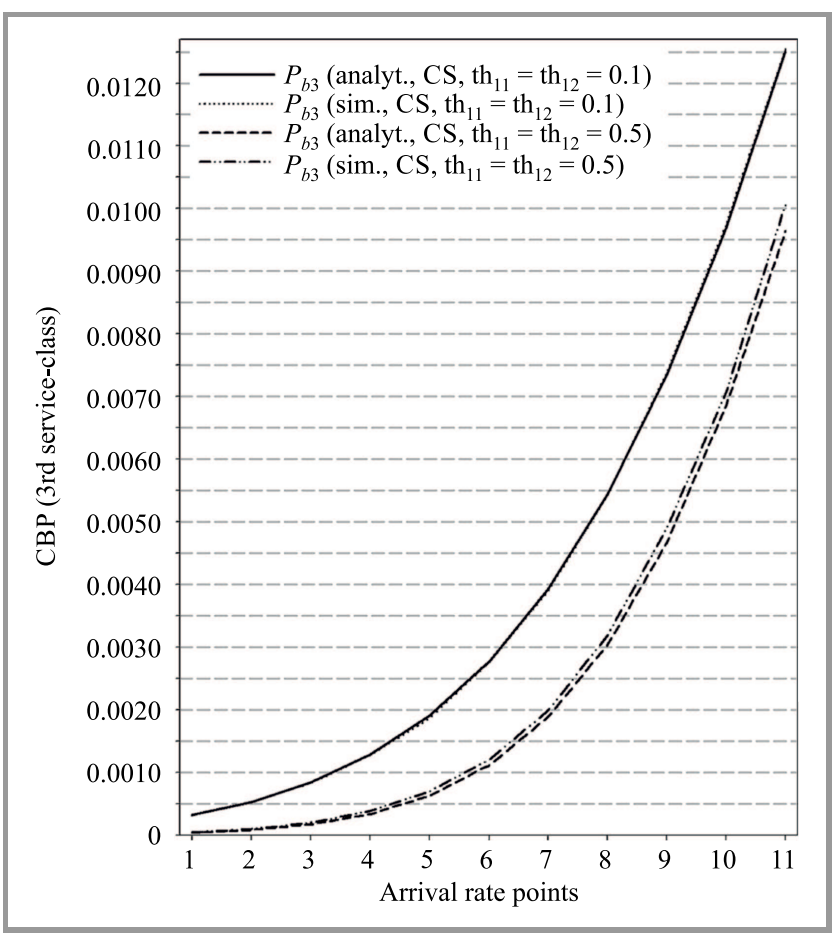

Fig. 4. CBP under the CS policy - 3rd service-class.

run. To account for a warm-up period, the first $5 \%$ of these generated calls are not considered in the CBP results.

As an application example, consider a system of two links of capacities $C_{1}=30$ and $C_{2}=25$ b.u., that accommodates $K=3$ service-classes whose calls require $b_{1}=1, b_{2}=2$ and $b_{3}=5$ b.u., respectively. For the 1 st link, let: $\lambda_{11}=$ 4.0, $\lambda_{12}=1.0, \lambda_{13}=0.5$. Similarly, for the 2 nd link, let: $\lambda_{21}=2.0, \lambda_{22}=1.0, \lambda_{23}=0.5$. We also assume that $\mu_{1}^{-1}=$ $\mu_{2}^{-1}=\mu_{3}^{-1}=1.0$.

We consider two different support thresholds: 1) $t h_{11}=$ $t h_{12}=0.1$ and 2) $t h_{11}=t h_{12}=0.5$. In both cases, we assume that the offloading thresholds do not alter and are equal to: $t h_{21}=t h_{22}=0.7$.

In the $\mathrm{x}$-axis of Figs. $2-4, \lambda_{11}$ and $\lambda_{21}$ increase in steps of 1.0 and 1.0, respectively. So, point 1 is: $\left(\lambda_{11}=4.0\right.$, $\left.\lambda_{12}=1.0, \lambda_{13}=0.5, \lambda_{21}=2.0, \lambda_{22}=1.0, \lambda_{23}=0.5\right)$ while point 11 is: $\left(\lambda_{11}=14.0, \lambda_{12}=1.0, \lambda_{13}=0.5, \lambda_{21}=12.0\right.$, $\left.\lambda_{22}=1.0, \lambda_{23}=0.5\right)$.

In Figs. 2-4, we present CBP in the 2EMLM for the three service-classes, respectively. Figures $2-4$ show that the analytical CBP results: a) are close to the simulation results and b) decrease as the support thresholds increase, an intuitively expected fact since both links cooperate with each other. Similar conclusions have been observed for systems of more than three service-classes but are not presented herein.

\section{Conclusion}

In this paper we propose a multirate loss model for a twolink loss system that accommodates Poisson arriving calls. A link can share a part of its capacity in order to support calls from the other link and vice versa. The proposed model does not have a PFS for the steady state distribution due to the existence of the offloading mechanism. However, we show that an approximate method does exist (based on a convolution algorithm) that provides quite satisfactory CBP results compared to simulation. As a future work, we intend to study this two-link system under the assumption that it serves different service-classes whose calls follow a quasi-random process, i.e. calls that are generated by a finite number of sources.

\section{Appendix - Tutorial Example}

Consider a system of two links with $C_{1}=6$ and $C_{2}=5$ b.u., that accommodates calls of a single service-class. Let $\lambda_{1}=4$ calls $/ \mathrm{min}, \lambda_{2}=2$ calls $/ \mathrm{min}$ and $\mu^{-1}=1 \mathrm{~min}$. The thresholds for this system are the following:

1st link $(l=1): t h_{11}=0.2, t h_{21}=0.7$,

2nd link $(l=2): t h_{12}=0.2, t h_{22}=0.7$.

Based on the thresholds' values we have:

\section{First link}

a) If $0 \leq j_{1}<\left\lfloor t h_{11} C_{1}\right\rfloor \Rightarrow 0 \leq j_{1}<1$ then the 1 st link is in a support mode of operation.

b) If $\left\lfloor t h_{11} C_{1}\right\rfloor \leq j_{1}<\left\lfloor t h_{12} C_{1}\right\rfloor \Rightarrow 1 \leq j_{1}<4$ then the 1 st link is in a normal mode of operation.

c) If $\left\lfloor t h_{21} C_{1}\right\rfloor \leq j_{1} \Rightarrow 4 \leq j_{1}$ then the 1 st link is in an offloading mode of operation.

\section{Second link}

a) If $0 \leq j_{2}<\left\lfloor t h_{12} C_{2}\right\rfloor \Rightarrow 0 \leq j_{2}<1$ then the 2nd link is in a support mode of operation.

b) If $\left\lfloor t h_{12} C_{2}\right\rfloor \leq j_{2}<\left\lfloor t h_{22} C_{2}\right\rfloor \Rightarrow 1 \leq j_{2}<3$ then the 2 nd link is in a normal mode of operation. 
c) If $\left\lfloor t h_{22} C_{2}\right\rfloor \leq j_{2} \Rightarrow 3 \leq j_{2}$ then the 2 nd link is in an offloading mode of operation.

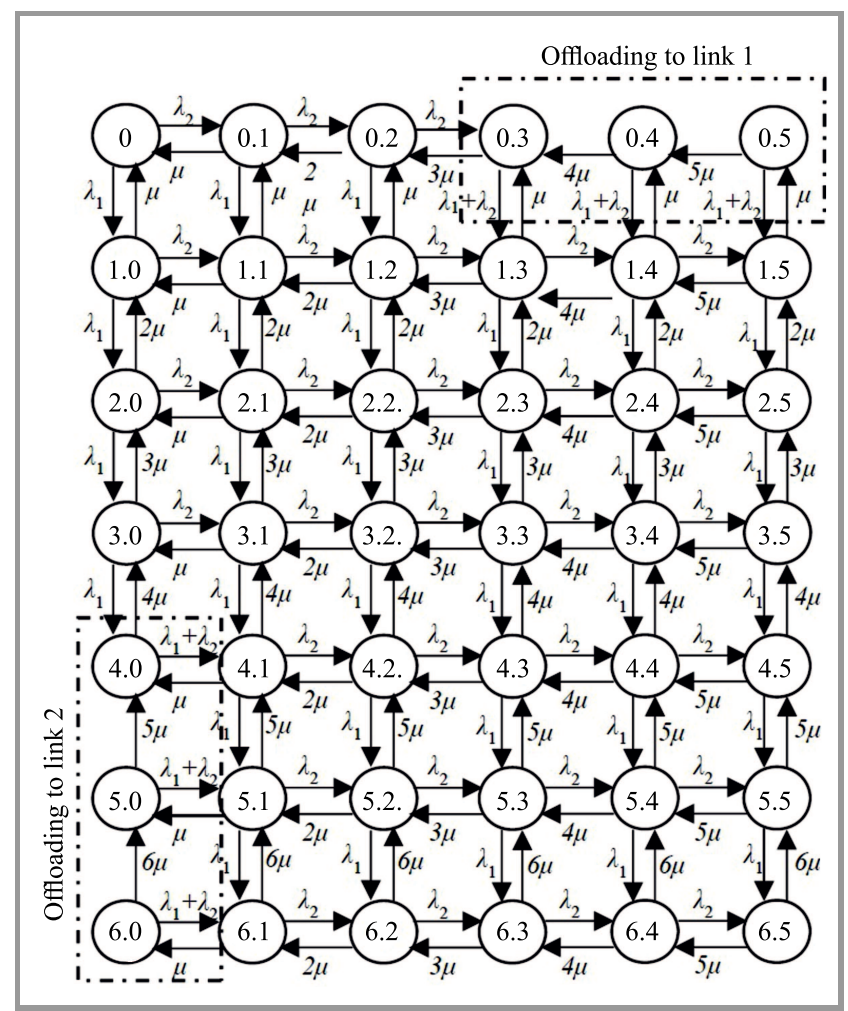

Fig. 5. State transition diagram of the tutorial example.

The state space of the system consists of 42 states of the form $\left(j_{1}, j_{2}\right)$, depicted in Fig. 5 together with the corresponding transition rates. To help a reader understand the state transition diagram of Fig. 5 and the offloading mechanism, assume that the system is in state $(0,2)$ when a new call arrives in the 2 nd link. Then, the call will be accepted in the 2 nd link and the new state will be $(0,3)$. If another new call arrives in the 2 nd link then the call will be offloaded to the 1 st link (and served by that link) and the new state will be $(1,3)$. If now, another call arrives in the 2nd link, then this call cannot be offloaded to the 1st link (since $j_{1}=1$ ) but it can be served by the 2 nd link due to bandwidth availability. In that case the new state will be $(1,4)$. A similar rationale exists when we consider call arrivals in the 1 st link and the states $(3,0),(4,0),(4,1)$ and $(5,1)$. Based on the solution of the $42 \mathrm{~GB}$ equations of Fig. 5, the CBP in the 1st and 2nd link is given by:

$$
\begin{aligned}
& P_{b_{1}}^{\prime}=\sum_{j_{2}=\left\lfloor t h_{12} C_{2}\right\rfloor} P\left(C_{1}, j_{2}\right)=\sum_{j_{2}=1}^{5} P\left(6, j_{2}\right)=0.10370, \\
& P_{b_{2}}^{\prime}=\sum_{j_{1}=\left\lfloor t h_{11} C_{1}\right\rfloor} P\left(j_{1}, C_{2}\right)=\sum_{j_{1}=1}^{6} P\left(j_{1}, 5\right)=0.03758 .
\end{aligned}
$$

On the same hand, the total blocking probability in the two-link system is determined by:

$$
P_{b}^{\prime}=\frac{\lambda_{1}}{\lambda_{1}+\lambda_{2}} P_{b_{1}}^{\prime}+\frac{\lambda_{2}}{\lambda_{1}+\lambda_{2}} P_{b_{2}}^{\prime} \stackrel{\lambda_{1}=4, \lambda_{2}=2}{=} 0.08166 .
$$

Based on the approximate method of link independence and Eqs. (6), (7), we have:

$P_{b_{1}}=P_{1}\left(C_{1}\right) P_{2}\left(j_{2} \geq\left\lfloor t h_{12} C_{2}\right\rfloor\right)=P_{1}(6) P_{2}\left(j_{2} \geq 1\right)=$ $0.11716 \times 0.862386 \Rightarrow P_{b}^{\prime}=0.10104$.

$P_{b_{2}}=P_{2}\left(C_{2}\right) P_{1}\left(j_{1} \geq\left\lfloor t h_{11} C_{1}\right\rfloor\right)=P_{2}(5) P_{1}\left(j_{1} \geq 1\right)=$ $0.03670 \times 0.979405 \Rightarrow P_{b}^{\prime}=0.03594$.

The total blocking probability in the two-link system is determined by:

$$
P_{b}=\frac{\lambda_{1}}{\lambda_{1}+\lambda_{2}} P_{b_{1}}+\frac{\lambda_{2}}{\lambda_{1}+\lambda_{2}} P_{b_{2}} \stackrel{\lambda_{1}=4, \lambda_{2}=2}{=} 0.07934
$$

The previous results reveal that the approximate method provides quite satisfactory results compared to the exact values, even in small tutorial examples.

\section{References}

[1] M. Stasiak, M. Głąbowski, A. Wiśniewski, and P. Zwierzykowski, Modeling, and Dimensioning of Mobile Networks: From GSM to LTE, Chichester, UK: Wiley \& Sons, 2010 (doi: 10.1002/9780470976036.ch3).

[2] M. Stasiak and M. Głąbowski, "A simple approximation of the link model with reservation by a one-dimensional Markov chain", Performance Evaluation, vol. 41, no. 2-3, pp. 195-208, 2000.

[3] I. Moscholios, M. Logothetis, and G. Kokkinakis, "Connection Dependent Threshold Model: A Generalization of the Erlang Multiple Rate Loss Model", Performance Evaluation, vol. 48, no. 1-4, pp. 177-200, 2002.

[4] M. Głąbowski and M. Stasiak, "Point-to-point blocking probability in switching networks with reservation", Annals of Telecommunications, vol. 57, no. 7-8, pp. 798-831, 2002.

[5] S. Rácz, B. Gerő, and G. Fodor, "Flow level performance analysis of a multi-service system supporting elastic, and adaptive services", Performance Evaluation, vol. 49, no. 1-4, pp. 451-469, 2002.

[6] M. Głąbowski and M. Stasiak, "Multi-rate model of the limited availability group with finite source population", in Proc. 10th AsiaPacific Conf. on Commun., Beijing, China, 2004.

[7] I. Moscholios and M. Logothetis, "Engset Multirate State-Dependent Loss Models with QoS Guarantee", Int. J. of Commun. Systems, vol. 19, no. 1, pp. 67-93, 2006.

[8] V. Vassilakis, I. Moscholios, and M. Logothetis, "Call-level performance modelling of elastic, and adaptive service-classes with finite population", IEICE Transact. on Commun., vol. E91-B, no. 1, pp. 151-163, 2008.

[9] Q. Huang, King-Tim Ko, and V. Iversen, "Approximation of loss calculation for hierarchical networks with multiservice overflows", in IEEE Transact. on Communic., vol. 56, no. 3, pp. 466-473, 2008.

[10] M. Stasiak, M. Sobieraj, J. Weissenberg, and P. Zwierzykowski, "Analytical Model of the Single Threshold Mechanism with Hysteresis for Multi-service Networks", IEICE Transact. on Commun., vol. E95-B, no. 1, pp. 120-132, 2012.

[11] I. Moscholios, J. Vardakas, M. Logothetis, and M. Koukias, "A Quasi-random Multirate Loss Model supporting Elastic, and Adaptive Traffic under the Bandwidth Reservation Policy", Int. J. on Advances in Networks and Services, vol. 6, no. 3-4, pp. 163-174, 2013.

[12] S. Yan, M. Razo, M. Tacca, and A. Fumagalli, "A Blocking Probability Estimator for the Multi-Application, and Multi-Resource Constraint Problem", in Proc. Int. Conf. Comp., Network., and Communic., Honolulu, HI, USA, 2014.

[13] I. Moscholios, M. Logothetis, J. Vardakas, and A. Boucouvalas, "Performance Metrics of a Multirate Resource Sharing Teletraffic Model with Finite Sources under both the Threshold and Bandwidth Reservation Policies", IET Networks, vol. 4, no. 3, pp. 195-208, 2015 . 
[14] Y. Huang, Z. Rosberg, K. Ko, and M. Zukerman, "Blocking Probability Approximations, and Bounds for Best-effort Calls in an Integrated Service System", IEEE Transact. on Commun., vol. 63, no. 12 , pp. 5014-5026, 2015.

[15] I. Moscholios, M. Logothetis, J. Vardakas, and A. Boucouvalas, "Congestion Probabilities of Elastic and Adaptive Calls in ErlangEngset Multirate Loss Models under the Threshold and Bandwidth Reservation Policies", Comput. Networks, vol. 92, no. 1, pp. 1-23, 2015.

[16] M. Głąbowski and M. Sobieraj, "Analytical modelling of multiservice switching networks with multiservice sources, and resource management mechanisms", accepted for publication in Telecoттиnication Systems, 2017 (doi:10.1007/s11235-017-0305-4).

[17] I. Widjaja and H. Roche, "Sizing X2 bandwidth for Inter-connected eNBs", in Proc. IEEE VTC Fall, Anchorage, AK, USA, 2009.

[18] M. Stasiak, P. Zwierzykowski, and D. Parniewicz, "Modelling of the WCDMA interface in the UMTS network with Soft Handoff Mechanism", in Proc. IEEE GLOBECOM, Honolulu, HI, USA, 2009.

[19] B. Renard, S. Elayoubi, and A. Simonian, "A dimensioning method for the LTE X2 interface", in Proc. IEEE WCNC, Shanghai, China, 2012.

[20] M. Stasiak, D. Parniewicz, and P. Zwierzykowski, "Traffic Engineering for Multicast Connections in Multiservice Cellular Network", IEEE Transactions on Industrial Informatics, vol. 9, no. 1, pp. 262-270, 2013.

[21] I. Moscholios, G. Kallos, M. Katsiva, V. Vassilakis, and M. Logothetis, "Call Blocking Probabilities in a W-CDMA cell with interference cancellation, and bandwidth reservation", in Proc. IEICE ICTF, Poznań, Poland, 2014.

[22] I. Moscholios, G. Kallos, V. Vassilakis, and M. Logothetis, "Congestion Probabilities in CDMA-based networks supporting batched Poisson input traffic", Wireless Person. Commun., vol. 79, no. 2, pp. 1163-1186, 2014

[23] M. Khedr, and R. Makki Hassan, "Opportunistic call admission control for wireless broadband cognitive networks", Wireless Networks, vol. 20 , no. 1 , pp. 105-114, 2014

[24] A. Machado de Medeiros, and M. Yacoub, "BlockOut: Blocking, and Outage in a Single Performance Measure", IEEE Transact. on Vehic. Technol., vol. 63, no. 7, pp. 3451-3456, 2014.

[25] V. Burger, M. Seufert, T. Hossfeld, and P. Tran-Gia, "Performance evaluation of backhaul bandwidth aggregation using a partial sharing scheme", Physic. Communic., vol. 19, pp. 135-144, 2016.

[26] I. Moscholios, V. Vassilakis, M. Logothetis, and A. Boucouvalas, "A Probabilistic Threshold-based Bandwidth Sharing Policy for Wireless Multirate Loss Networks", IEEE Wireless Commun. Let., vol. 5, no. 3, pp. 304-307, 2016.

[27] V. Vassilakis, I. Moscholios, and M. Logothetis, "Uplink Blocking Probabilities in Priority-Based Cellular CDMA Networks with Finite Source Population", IEICE Transact. on Commun., vol. E99-B, no. 6, pp. 1302-1309, 2016.

[28] V. Vassilakis, I. Moscholios, and M. Logothetis, "Quality of Service Differentiation of Elastic, and Adaptive Services in CDMA Networks: A Mathematical Modelling Approach", accepted for publication in Wireless Networks, 2017 (doi:10.1007/s11276-016-1411-z).

[29] Z. Wang, P. Mathiopoulos, and R. Schober, "Performance Analysis and Improvement Methods for Channel Resource Management Strategies of LEO-MSS with Multiparty Traffic", IEEE Transact. on Vehic. Technol., vol. 57, no. 6, pp. 3832-3842, 2008.

[30] D. Yiltas and A. Zaim, "Evaluation of call blocking probabilities in LEO satellite networks", Int. J. of Satel. Communic., vol. 27, no. 2, pp. 103-115, 2009.

[31] Z. Wang, P. Mathiopoulos, and R. Schober, "Channel partitioning policies for multi-class traffic in LEO-MSS", IEEE Trans. on Aerosp., and Electr. Sys., vol. 45, no. 4, pp. 1320-1334, 2009.

[32] J. Vardakas, I. Moscholios, M. Logothetis, and V. Stylianakis, "An Analytical Approach for Dynamic Wavelength Allocation in WDMTDMA PONs Servicing ON-OFF Traffic", IEEE/OSA J. of Optic. Communic. and Network., vol. 3, no. 4, pp. 347-358, 2011.

[33] Y. Deng and P. Prucnal, "Performance analysis of heterogeneous optical CDMA networks with bursty traffic, and variable power control", IEEE/OSA J. of Optic. Commun. and Network., vol. 3, no. 6, pp. 487-492, 2011.
[34] J. Vardakas, I. Moscholios, M. Logothetis, and V. Stylianakis, "On Code reservation in Multi-rate OCDMA Passive Optical Networks", in Proc. 8th IEEE Int. Symp. on Commun. Sys., Networks \& Digit. Signal Process. CSNDSP, Poznań, Poland, 2012.

[35] J. Vardakas, I. Moscholios, M. Logothetis, and V. Stylianakis, "Performance Analysis of OCDMA PONs Supporting Multi-Rate Bursty Traffic", IEEE Transact. on Commun., vol. 61, no. 8, pp. 3374-3384, 2013.

[36] V. Casares-Giner, "Some teletraffic issues in optical burst switching with burst segmentation”, Electr. Let., vol. 52, no. 11, pp. 941-943, 2016.

[37] Y. Guan, H. Jiang, M. Gao, S. Bose, and G. Shen, "Migrating Elastic Optical Networks from Standard Single-Mode Fibers to Ultra-Low Loss Fibers: Strategies, and Benefits", in Proc. Optic. Fiber Communic. Conf., Los Angeles, CA, USA, 2017.

[38] S. Pantelis, I. Moscholios, and S. Papadopoulos, "Call-level evaluation of a two-link single rate loss model for Poisson traffic", in Proc. IEICE ICTF, Poznań, Poland, 2017.

[39] L. Mamatas, I. Psaras, and G. Pavlou, "Incentives, and Algorithms for Broadband Access Sharing", in Proc. ACM SIGCOMM Workshop on Home Networks, New Delhi, India, 2010.

[40] I. Psaras and L. Mamatas, "On demand connectivity sharing: Queuing management, and load balancing for user-provided networks", Comput. Networks, vol. 55, no. 2, pp. 399-414, 2011.

[41] BeWi-Fi, October 2017 [Online]. Available: http://www.tid.es/research/areas/beWi-Fi

[42] J. Kaufman, "Blocking in a shared resource environment", IEEE Transact. on Communic., vol. 29, no. 10, pp. 1474-1481, 1981.

[43] J. Roberts, "A service system with heterogeneous user requirements", in: G. Pujolle (Ed.), Performance of Data Communications systems, and their applications, Amsterdam, North Holland, the Netherlands, pp. 423-431, 1981.

[44] V. Iversen, "The exact evaluation of multi-service loss system with access control", Teleteknik, vol. 31, no. 2, pp. 56-61, 1987.

[45] D. Tsang and K. Ross, "Algorithms to determine exact blocking probabilities for multirate tree networks", IEEE Transact. on Commun., vol. 38, no. 8, pp. 1266-1271, 1990 .

[46] Q. Huang, K. Ko, and V. Iversen, "Approximation of loss calculation for hierarchical networks with multiservice overflows", IEEE Transact. on Commun., vol. 56, no. 3, pp. 466-473, 2008.

[47] M. Głąbowski, A. Kaliszan, and M. Stasiak, "Asymmetric convolution algorithm for full-availability group with bandwidth reservation”, in Proc. Asia-Pacific Conf. on Commun., Busan, South Korea, 2006.

[48] M. Głąbowski, A. Kaliszan, and M. Stasiak, "Asymmetric convolution algorithm for blocking probability calculation in full-availability group with bandwidth reservation", IET Circuits, Devices \& Systems, vol. 2, no. 1, pp. 87-94, 2008.

[49] M. Głabowski, A. Kaliszan, and M. Stasiak, "Two-dimensional convolution algorithm for modelling multiservice networks with overflow traffic", Mathemat. Problems in Engin., vol. 2013, Article ID 852082, 18 pages.

[50] I. Moscholios, "Call blocking probabilities in an Erlang Multirate Loss Model under a State-dependent Threshold Policy", in Proc. of IEICE Infor. and Communic. Technol. Forum ICTF, Patras, Greece, 2016.

[51] S. Hanczewski, A. Kaliszan, and M. Stasiak, "Convolution model of a queueing system with the cFIFO service discipline", Mob. Infor. Sys., vol. 2016, Article ID 2185714, 15 pages.

[52] S. Sagkriotis and I. Moscholios, "Evaluation of convolution algorithms in the Erlang Multirate Loss Model under the bandwidth reservation policy", in Proc. IEICE ICTF, Poznań, Poland, 2017.

[53] I. Moscholios, V. Vassilakis, M. Logothetis, and A. Boucouvalas, "State-dependent Bandwidth Sharing Policies for Wireless Multirate Loss Networks", IEEE Transact. on Wireless Commun., vol. 16, no. 8, pp. 5481-5497, 2017.

[54] Simscript III, October 2017 [Online]. Available: http://www.simscript.com 


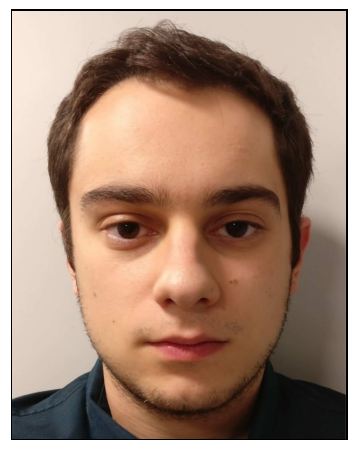

Stefanos G. Sagkriotis received his B.Sc. in Informatics and Telecommunications from the University of Peloponnese, Tripolis, Greece. Currently he is an M.Sc. student at the School of Computing Science, University of Glasgow, Glasgow, United Kingdom. His research interests include teletraffic engineering and network

performance evaluation.

E-mail: : 2353747s@student.gla.ac.uk

School of Computing Science

University of Glasgow

Glasgow, United Kingdom

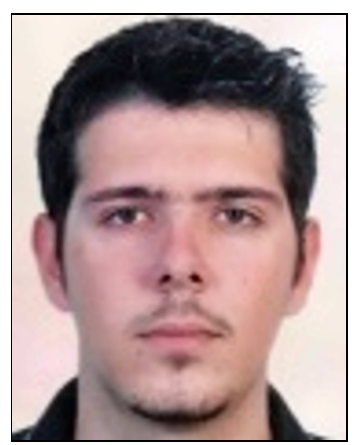

Spiridon K. Pantelis is an undergraduate student in the Department of Informatics and Telecommunications, University of Peloponnese, Greece. His research interests include teletraffic engineering and network performance evaluation.

E-mail: tst12081@uop.gr

Department of Informatics and Telecommunications

University of Peloponnese

Tripolis, Greece

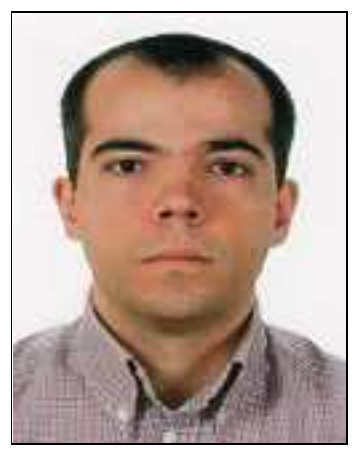

Ioannis D. Moscholios received his D.Eng. degree in Electrical and Computer Engineering from the University of Patras, Patras, Greece, in 1999, M.Sc. degree in Spacecraft Technology and Satellite Communications from the University College London, UK, in 2000 and Ph.D. degree in Electrical and Computer Engineering from the University of Patras, in 2005. From 2005 to 2009 he was a Research Associate at the Wire
Communications Laboratory, Department of Electrical and Computer Engineering, University of Patras. From 2009 to 2013 he was a Lecturer in the Department of Telecommunications Science and Technology, University of Peloponnese, Tripolis, Greece. Currently, he is an Assistant Professor in the Department of Informatics and Telecommunications, University of Peloponnese. His research interests include teletraffic engineering, simulation and performance analysis of communication networks. He is an IARIA Fellow and a member of the Technical Chamber of Greece. E-mail: idm@uop.gr Department of Informatics and Telecommunications University of Peloponnese Tripolis, Greece

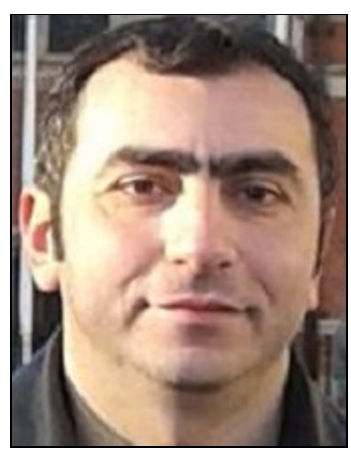

Vassilios G. Vassilakis is a lecturer in the Department of Computer Science at the University of York, UK. He received his Ph.D. degree in Electrical and Computer Engineering from the University of $\mathrm{Pa}$ tras, Greece, in 2011. From 2011 to 2013 , he was with the Network Convergence Laboratory, University of Essex, where he conducted research on information-centric networking and network security, and contributed to the EC FP7 PURSUIT project. In 2013, he joined the Institute for Communication Systems, University of Surrey, and conducted research on $5 \mathrm{G}$ wireless networks. After that, he was with the Computer Laboratory, University of Cambridge, where he conducted research on future Internet technologies and contributed to the EC H2020 RIFE project. In 2015, he joined the Secure and Dependable Software Systems Research Cluster at the University of Brighton, where he conducted research on $5 \mathrm{G}$ network security and contributed to the EC H2020 SESAME project. His main research interests are in the areas of $5 \mathrm{G}$ wireless and mobile networks, future Internet technologies, network security, software-defined networks, and IoT. He has published over 80 journal/conference papers. E-mail:vv274@cl.cam.ac.uk

Department of Computer Science University of York York, United Kingdom 